Title of article

\title{
A New Design Approach for Control Circuits of Pipelined Single-Flux-Quantum Microprocessors
}

Authors and addresses

Y. Yamanashi ${ }^{1}$, A. Akimoto ${ }^{1}$, N. Yoshikawa ${ }^{1}$, M. Tanaka ${ }^{2}$, T. Kawamoto ${ }^{2}$, Y. Kamiya ${ }^{2}$, A. Fujimaki $^{2}, \mathrm{H}$. Terai ${ }^{3}$ and S. Yorozu ${ }^{4}$

${ }^{1}$ Department of Electrical and Computer Engineering, Yokohama National University, Tokiwadai 79-5, Hodogaya, Yokohama, 240-8501, Japan

${ }^{2}$ Department of Quantum Engineering, Nagoya University, Furo-cho, Chikusa, Nagoya, 464-8603, Japan

${ }^{3} \mathrm{NiCT}$, 588-2 Iwaoka, Nishi-ku, Kobe 651-2492, Japan

${ }^{4}$ ISTEC-SRL, 34 Miyukigaoka, Tsukuba 305-8501, Japan

Corresponding author

Yuki Yamanashi

Department of Electrical and Computer Engineering, Yokohama National University, Tokiwadai 79-5, Hodogaya, Yokohama, 240-8501, Japan.

Phone: +81-45-339-4269

Fax: +81-45-338-1157

E-mail: yamanasi@yoshilab.dnj.ynu.ac.jp

Short title

A New Design Approach for Control Circuits of Pipelined SFQ Microprocessors

Classification numbers

PACS: $85.25 . \mathrm{Hv}$

The abstract

A novel design method for controllers of pipelined microprocessors using single flux quantum (SFQ) logic has been proposed. The proposed design approach is based on one hot encoding and very suitable for designing a finite state machine using SFQ logic circuits, where 
each internal state of the microprocessor is represented by a flip-flop. In this approach decoding of the internal state can be performed instantaneously, in contrast to the conventional method using a binary state register. Moreover, pipelining is effectively implemented without increasing the circuit size because no pipeline registers are required in the one hot encoding. By using this method, we have designed a controller of our new SFQ microprocessors, which employs pipelining. The number of Josephson junctions of the newly designed controller is 1067, while the previous version without pipelining, contains 1721 Josephson junctions. These results indicate that the proposed design approach is very effective for pipelined SFQ microprocessors. We have implemented a new controller using the NEC $2.5 \mathrm{kA} / \mathrm{cm}^{2} \mathrm{Nb}$ standard process and confirmed its correct operation experimentally. 


\section{Introduction}

Single flux quantum (SFQ) logic circuits are very promising technology for future high-end information processing systems because of their high-speed and low-power operation [1]. We have been developing microprocessors using SFQ circuits. So far, we have designed and demonstrated several small SFQ microprocessors named CORE1 $\alpha$. CORE1 $\alpha$ is a simple 8-bit microprocessor based on our complexity-reduced (CORE) concept [2], where high-throughput of SFQ circuits is fully utilized. We have demonstrated complete operations of the first prototype microprocessor [3], its improved version using passive transmission lines (PTL) [4], and a version integrated with a 4-byte memory. The demonstrated maximum performance of CORE1 $\alpha$ was 240 MIPS (million instructions per second) [4].

To enhance the performance of microprocessors further, introduction of pipelining is very effective because multiple instructions can be executed in one clock cycle in this technique. However, a controller, which handles all circuit components of the microprocessor by providing control signals, becomes very complicated in a conventional design method [5] because a lot of pipeline registers are required to keep the control information for each pipeline stage.

In this study, we have considered a new design approach suitable for designing a controller of pipelined SFQ microprocessors based on one hot encoding. The proposed approach requires no pipeline register, therefore, a large number of pipeline stages can be easily made without hardware costs. We have designed and implemented controllers of our new pipelined SFQ microprocessors, named CORE1 $\beta \square$, by using this method.

\section{Pipeline Architecture}

Pipelining is a technique in which multiple instructions are overlapped in execution. This technique is indispensable for enhancing the performance of present microprocessors. Fig. 1 shows a datapath and control lines of a conventional five-stage pipelined microprocessor based on CMOS LSI technology [6]. In order to implement pipelining, each execution of instructions is divided into several stages, called pipeline stages. In the example of Fig. 1, the pipeline stage consists of five stages: instruction fetch (IF), instruction decode (ID), execution (EX), data memory access (MEM) and write back (WB), where the execution of an instruction takes five steps to complete.

To control the pipelined microprocessor, a lot of pipeline registers, which keep pipeline control information, are needed in a conventional design approach as shown in Fig. 1. This is because the pipeline control information must be kept until the completion of the instruction. Besides the controller becomes complicated with increase of the number of the pipeline stages. The conventional design approach is, therefore, not suitable for the SFQ microprocessor because its pipeline stage usually becomes very deep. For example bit-level pipelining is used in 
an extreme case [7].

\section{A New Design Approach of a Controller of Pipelined SFQ Microprocessors}

A controller for microprocessors is classified into a finite-state machine or a sequential logic circuit. A block diagram of the sequential logic circuit for the controller using a conventional design approach is shown in Fig. 2, which is composed of a combinational logic circuit and a binary state register. In the conventional design, the internal states of the sequential logic are encoded to a binary code and stored in the binary state register. This method is useful to decrease the size of the state register. However, the latency of the circuit is fairly large because the internal state stored in the binary state register has to be decoded every clock cycle. Besides, in the controller for the pipelined microprocessor, a number of state registers are necessary with increasing the number of the pipeline stages, resulting in large complexity and latency of the controller.

An alternative implementation method of the sequential logic circuit is to use one hot encoding as shown in Fig. 3. The internal state of the sequential logic is generally denoted by a state transition diagram. In the sequential circuit using one hot encoding, its state transition diagram is directory made by a network of 1-bit flip-flops, each of which corresponds to one state in the state transition diagram. In this approach, if a number of the internal state of the sequential logic is ten for example, ten flip-flops are used, and only one flip-flop has datum "1" to represent the current state of the circuit. It is apparent that the disadvantage of this approach is the number of flip-flops becomes considerably large with the increase of the number of internal states. However the latency of the circuit is quite small because decoding time of the internal state is eliminated. This feature compensates for the disadvantage of the large circuit size. It should be noted here that this approach is very suitable for SFQ logic circuits because implementation costs of the flip-flop is cheep in SFQ logic circuits. The one hot encoding is also effective in implementing the pipelined microprocessor. Because each flip-flop corresponds to the internal state of the sequential logic, we can introduce pipelining to a controller by simply adding datum " 1 " to the flip-flops in the network, where the number of the datum "1" corresponds to the number of the pipeline stages.

\section{Design and Test of a Controller of Pipelined SFQ Microprocessors}

The CORE1 $\beta$ microprocessor is an 8-bit bit-serial microprocessor based on forwarding architecture, where the cascade connection of several ALUs enhances the performance of the datapath by executing several ALU operations during one instruction cycle [8]. The CORE1 $\beta$ can perform eight instructions composed of a register operation (R-type), data transfers (Load, Sore), branches (Jump, Branch equal zero, Branch not equal zero), no operation (Nop), and Halt, 
which are determined by a 4-bit opcode. We have designed several versions of CORE1 $\beta$ microprocessors. The first version of the microprocessor, CORE1 $\beta$ ver.1, does not use pipeline architecture. In CORE1 $\beta$ ver.1 the execution of one instruction consists of seven phases, each of which is advanced by applying the system clock to the controller. The system clock frequency designed is $1 \mathrm{GHz}$, so each instruction is executed every $7 \mathrm{~ns}$. The second and third version of microprocessors, CORE1 $\beta$ ver. 2 and ver.3, employ the pipeline architecture with two and four pipeline stages, respectively. Fig. 4 shows a state transition diagram of the CORE1 $\beta$ ver.2, which has 29 internal states composed of six phases. In the phase 2 the state is branched into one of eight states according to the 4-bit opcode.

We have designed and implemented controllers for pipelined SFQ microprocessors using the proposed new design method, where we used the NEC $2.5 \mathrm{kA} / \mathrm{cm}^{2}$ standard process [9] and the CONNECT cell library [10]. Fig. 5 shows a circuit schematic of the controller of the CORE1 $\beta$ ver.2. In this design SFQ delay flip-flops are used to represent the state in the state transition table. Some of them are indicated by open circles in the figure. In table 1 we compare the features of controllers of our three versions of microprocessors. It should be noted that we did not use the one hot encoding in designing the controller of the CORE1 $\beta$ ver.1. As shown in table 1 , the area and the number of Josephson junctions of the controller for the CORE1 $\beta$ ver. 2 and ver.3 are reduced remarkably compared with that of the CORE1 $\beta$ ver.1, even though the pipeline architecture is employed in the latter two versions. One can also see that the performance of the microprocessors is enhanced dramatically as a result of the introduction of the pipelining.

We have tested the operation of the controller of the CORE1 $\beta$ ver.2 at low speed. Fig. 6 shows examples of test results. We have confirmed the correct operation of every instruction of the microprocessor experimentally. The experimental results show that the dc bias margin of the controloer is $-5 \% \sim+5 \%$.

\section{Conclusion}

We have proposed a new design method for SFQ sequential logic circuits based on one hot encoding. The proposed design approach is suitable for designing controllers of pipelined microprocessors. We have designed controllers of our pipelined SFQ microprocessors using the proposed design method and shown that the number of Josephson junctions and the circuit area are reduced considerably compared with our previous controller design using the conventional design method. The results indicate that the proposed design approach is very effective for designing pipelined SFQ microprocessors. 


\section{References}

[1] Likharev K K and Semenov V K, 1991 RSFQ logic/memory family: a new Josephson-junction technology for sub-teraherta-clock-digital systems IEEE Trans. Appl. Supercond. 1 3-28

[2] Fujimaki A, Takai Y and Yoshikawa N 2002 High-end server based on complexity-reduced architecture for superconductor technology IEICE Trans. Electron. 85 612-6

[3] Tanaka M, Matsuzaki F, Kondo T, Nakajima N, Yamanashi Y, Terai H, Yorozu S, Yoshikawa N, Fjimaki A and Hayakawa H, 2004 Demonstration of a prototype of the microprocessor based on the single-flux-quantum logic Microprocessor", Technical Digest of IEEE International

Solid-State Circuits Conference (ISSCC) 2004, Feb. 2004

[4] Tanaka M, Kondo T, Nakajima N, Kawamoto T, Yamanashi Y, Kamiya Y, Akimoto A, Fujimaki A, Hayakawa H, Yoshikawa N, Terai H, Hashimoto Y and Yorozu S 2005 Demonstration of a Single-Flux-Quantum Microprocessor Using Passive Transmission Lines IEEE Trans. Appl. Supercond. 15 400-4

[5] Nakajima N, Matsuzaki F, Yamanashi Y, Yoshikawa N, Tanaka M, Kondo T, Fujimaki A, Terai H and Yorozu S 2004 Design and implementation of circuit components of the SFQ microprocessor, CORE1 Supercond. Sci. Technol. 17 301-7

[6] Hennessy J and Patterson D A 2003 Computer Architecture, A Quantitative Approach, Third Edition Morgan Kaufmann Publishers

[7] Dorojevets M, Bunyk P and Zinoviev D, 2001 FLUX chip design of 20-GHz 16-bit ultrapipelined RSFQ processor prototype based on $1.75-\mu \mathrm{m}$ LTS technology IEEE Trans. Appl. Supercond. 11 326-32

[8] Tanaka M, Kawamoto T, Yamanashi Y, Kamiya Y, Akimoto A, Fujiwara K, Fujimaki A, Yoshikawa N, Terai H and Yorozu S 2005 Design of a pipelined 8-bit-serial single-flux-quantum microprocessor with multiple ALUs 10th International Superconductive Electronics Conference [9] Nagasawa S, Hashimoto Y, Numata H and Tahara S 2003 A 380 ps, $9.5 \mathrm{~mW}$ Josephson 4-kbit RAM operated at a high bit yield IEEE Trans. Appl. Supercond. 5 2447-52

[10] Yorozu S, Kameda Y, Terai H, Fujimaki A, Yamada H and Tahara S 2002 A single flux quantum standard logic cell library Physica C 378-381 1471-4 
Figure captions

Fig. 1. Example of a datapath with control lines of a conventional five-stage pipelined microprocessor. Dashed lines represent control signals. In the implementation of pipelining, many pipeline registers are required to keep control information for each stage.

Fig. 2. A block diagram of a sequential logic circuit using a conventional binary register.

Fig. 3. A block diagram of a sequential logic circuit using one hot encoding.

Fig. 4. A state transition diagram of the CORE1 $\beta 2$ ver.2 microprocessor.

Fig. 5. A circuit schematic of the controller of the pipelined SFQ microprocessor,

CORE1 $\beta$ ver.2. Open circles indicate positions of delay flip-flops corresponding to the internal states of the microprocessor.

Fig. 6. Low-speed test results of the controller of the CORE1 $\beta$ ver. 2 microprocessor when instructions, Halt (a) and R_type (b), were executed. The upper six waveforms are input signals, where the rising edges correspond to the input SFQ pulses. The lower three waveforms are output signals, where transitions are the output of SFQ pulses. The scales of vertical axis are 2 $\mathrm{V} / \mathrm{div}$ for input signals and $200 \mu \mathrm{V} / \mathrm{div}$ for output signals, respectively. After the initialization of the internal state of the control circuit (1), a 4-bit opcode is input from LSB (the least significant bit) (2). Then the instruction is decoded by inputting six clock pulses for the controller (3), and the control signals are read out from the controller by inputting a "Read" signal to an on-chip read-out shift-register (4). A HLT_trg output is obtained when the instruction, HLT, is executed (a). We can also confirm correct outputs of many control signals for the circuit components of the microprocessor when the R_type instruction is executed (b). 


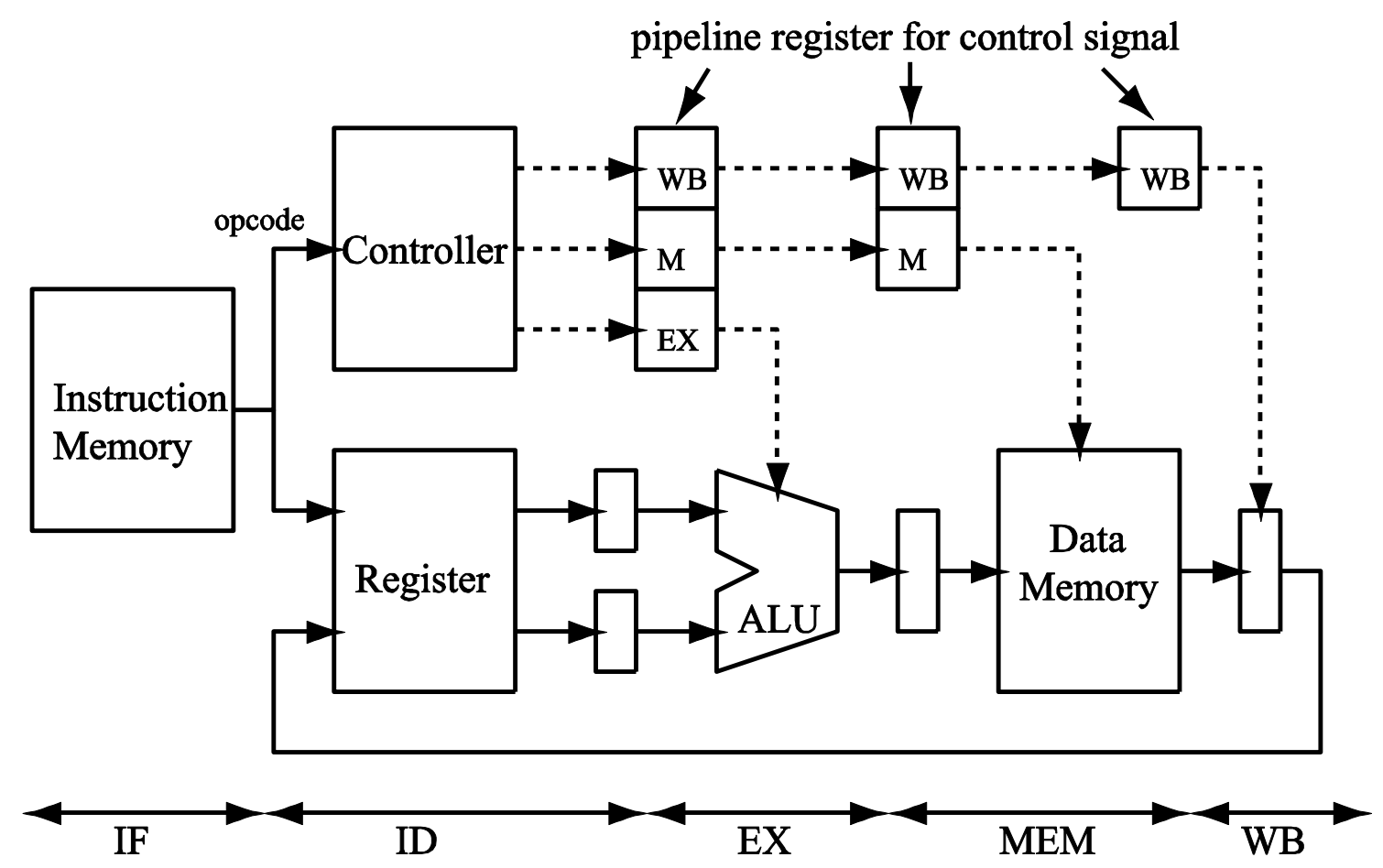

Fig. 1 


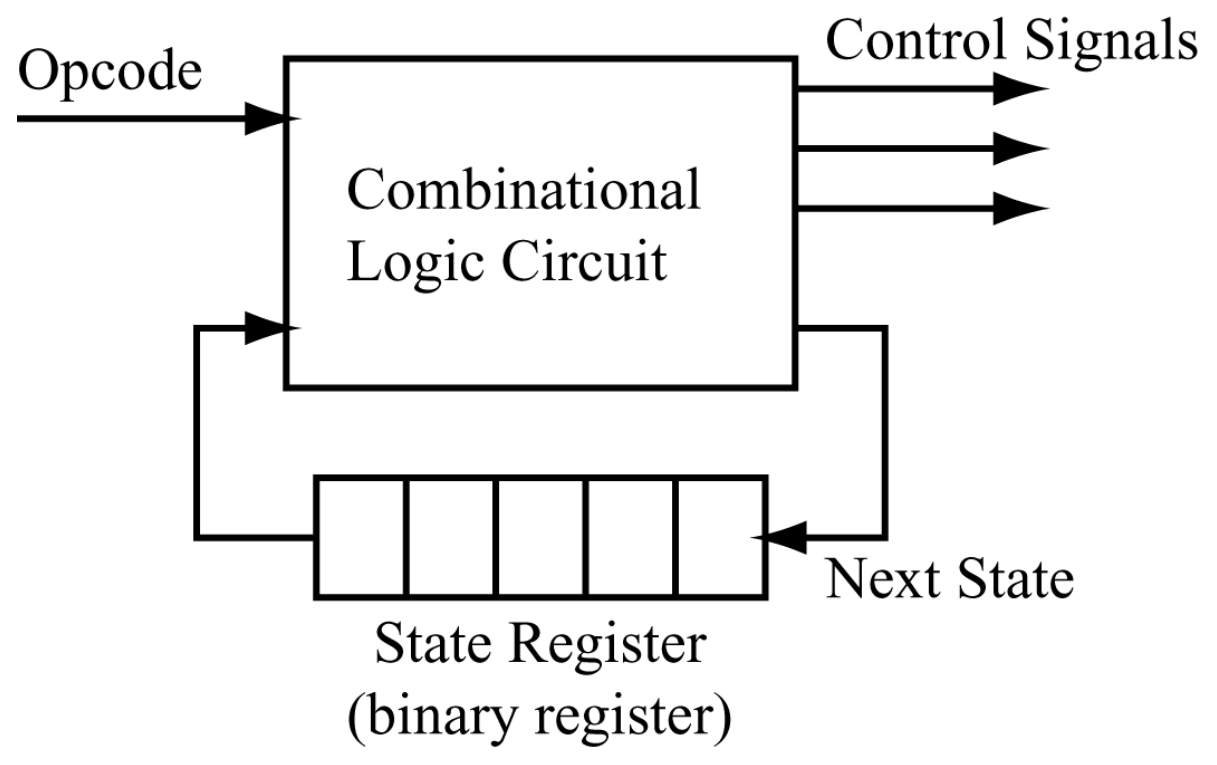

Fig. 2 


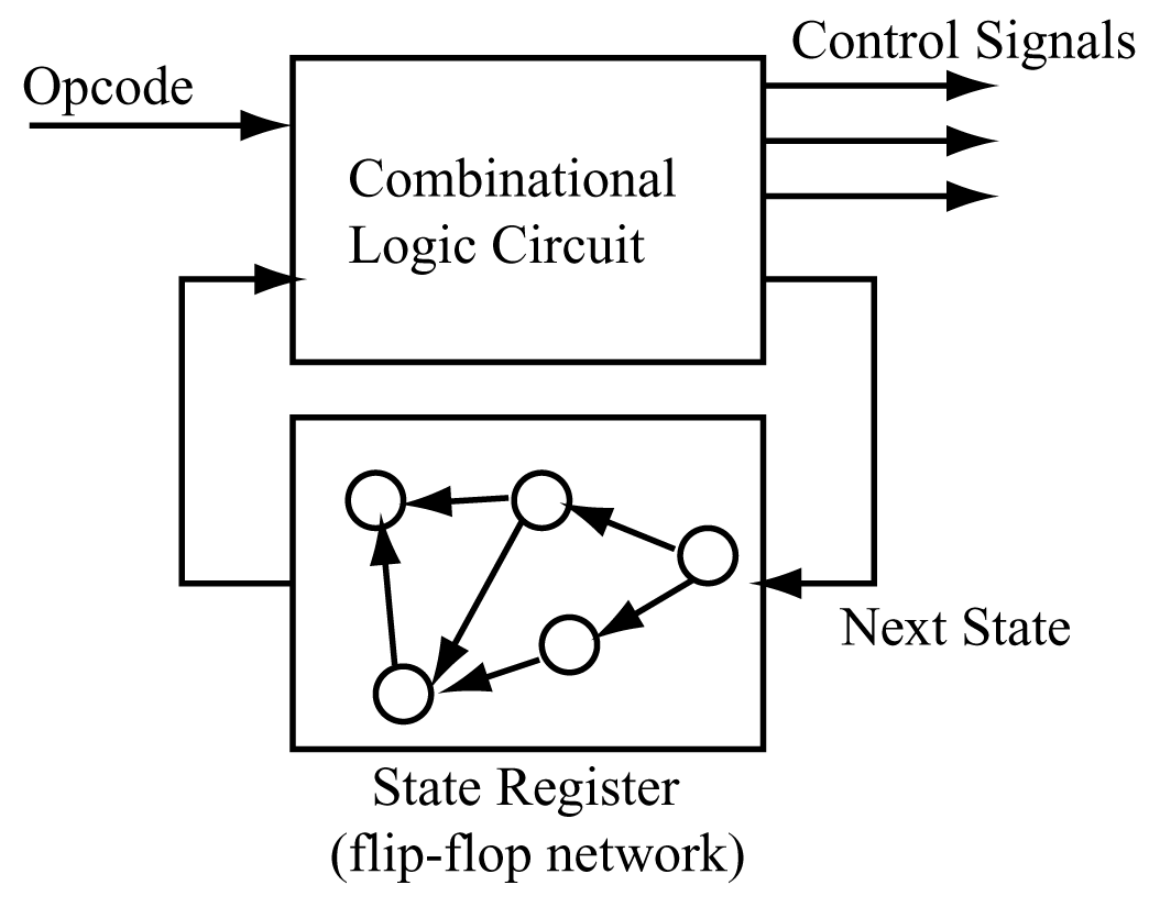

Fig. 3 


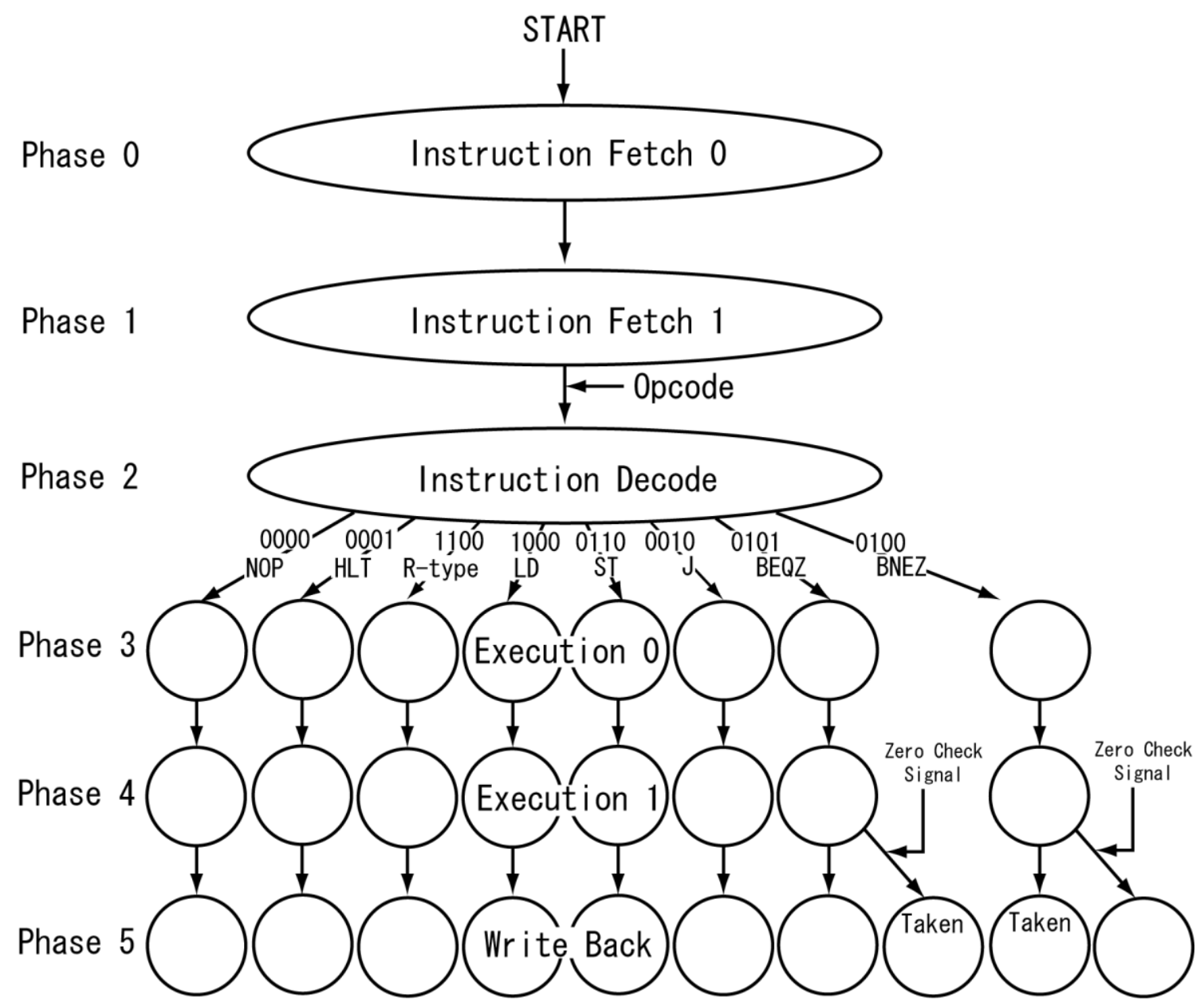

Fig. 4 


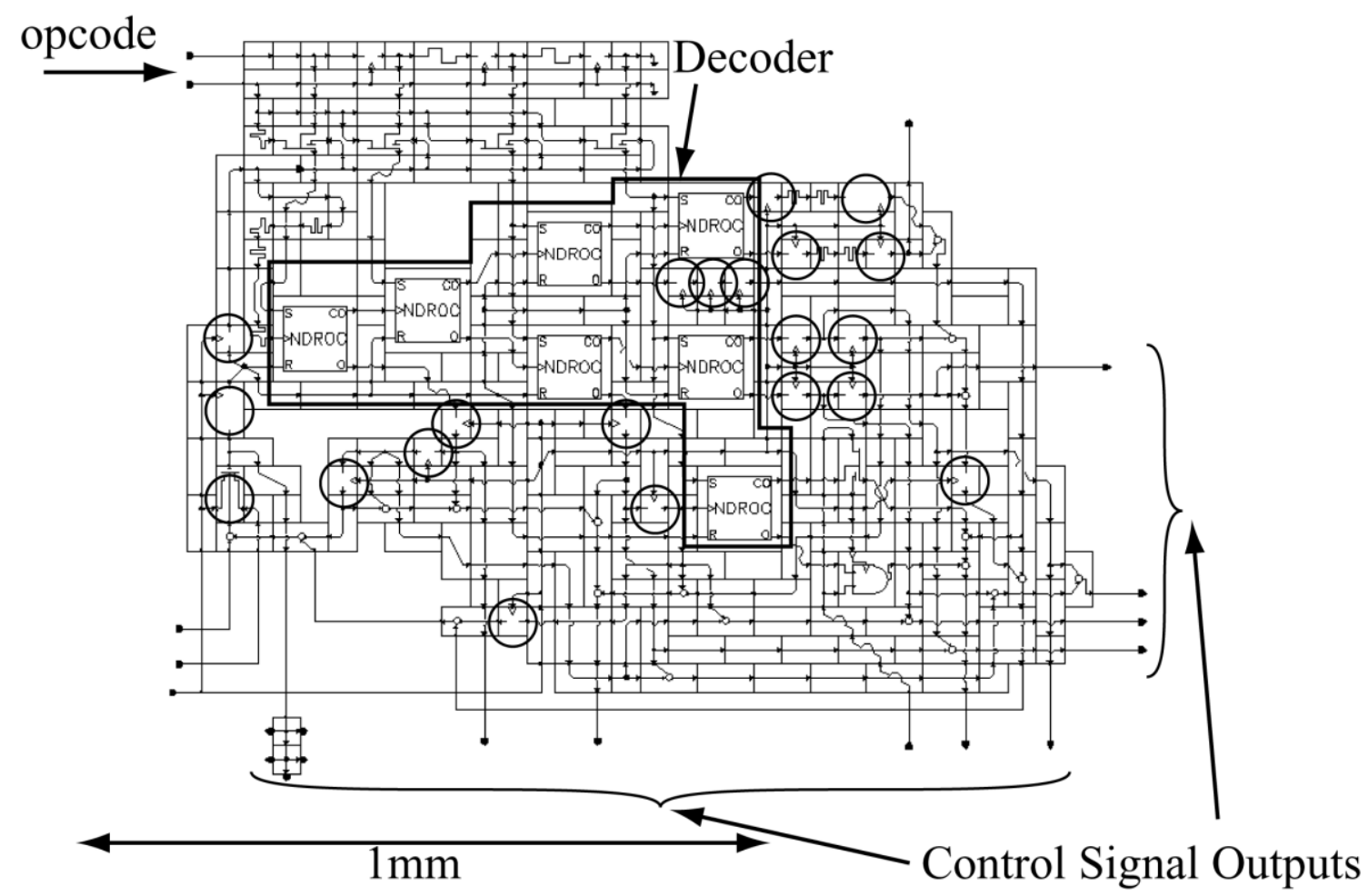

Fig. 5 


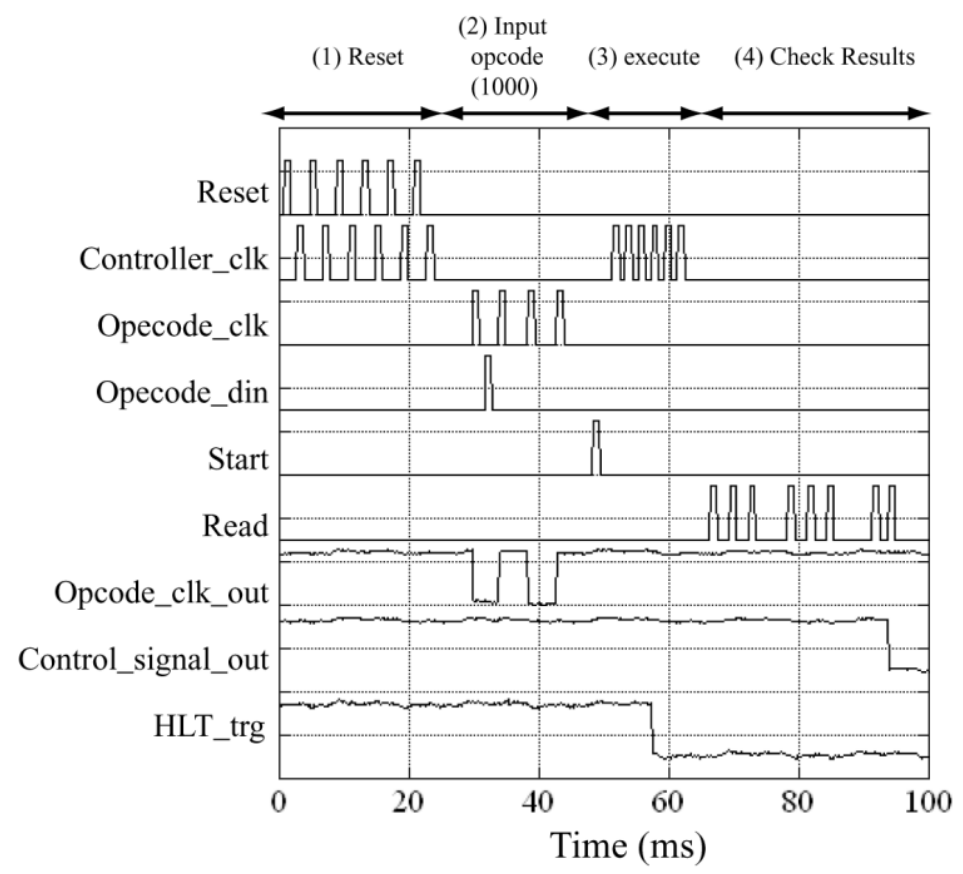

(a)

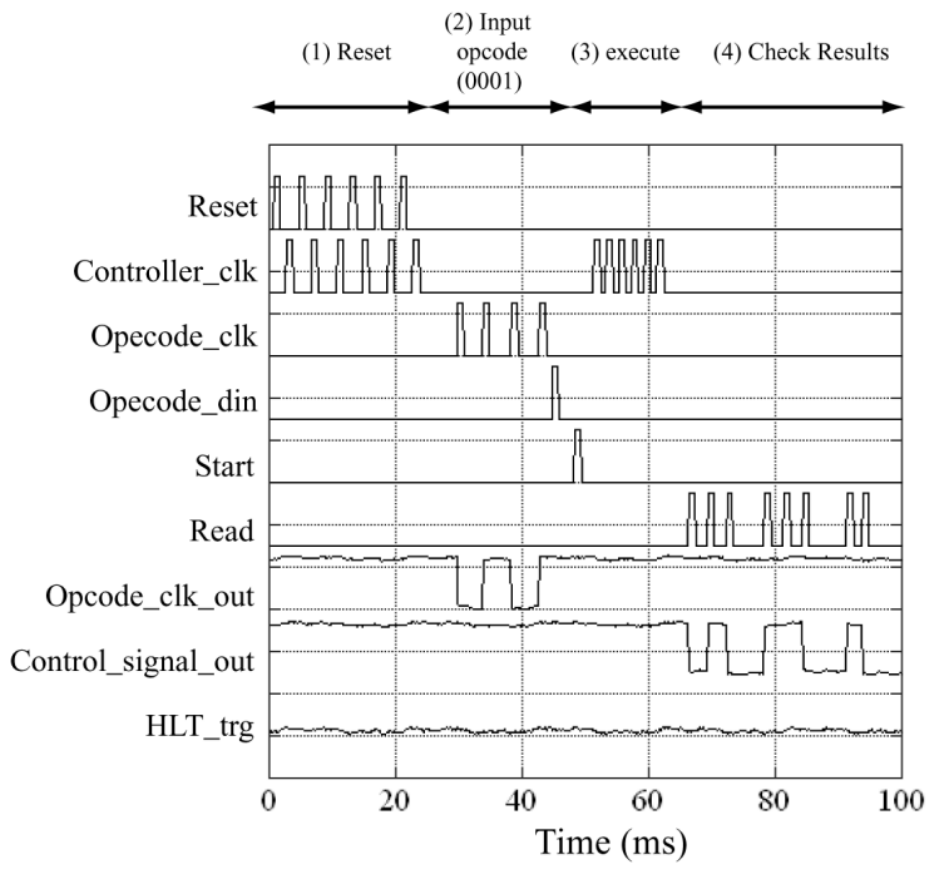

(b)

Fig. 6 
TABLE I

COMPARISON OF THREE CONTROL CIRCUITS

\begin{tabular}{cccc}
\hline \hline Version & CORE1 $\beta$ ver.1 & CORE1 $\beta$ ver.2 & CORE1 $\beta$ ver.3 \\
\# of Phases & 7 & 6 & 8 \\
Pipeline stages & No pipelining & 2 & 4 \\
Performance (MOPS) & 286 & 667 & 1000 \\
One-hot Encoding & Not employed & Employed & Employed \\
\# of Josephson Junctions & 1721 & 1339 & 1067 \\
Area (mm) & $1.28 \times 1.32$ & $0.92 \times 1.32$ & $0.92 \times 0.92$ \\
\# of Logic Cells & 48 & 48 & 44 \\
\hline \hline
\end{tabular}

